\title{
ON THE DEVELOPMENT OF PLASTIC HINGES IN RIGID-PLASTIC BEAMS*
}

\author{
BY M. G. SALVADORI AND F. DIMAGGIO (Columbia L'niversity)
}

Synopsis. A study is made of the derelopment of plastic hinges in free-free, rigidplastic beams acted upon hy certain types of distributed, dynamic loads, characterized hy a concentration parameter $c$, whose value varies between zero and infinity as the load distribution varies from a uniform to a concentrated load.

It is found that as the load intensity increases a plastic hinge is first developed at the center of the beam, whatever the value of the concentration parameter.

As the load intensity increases beyond this value, the development of successive hinges depends upon the parameter $c$ : two additional hinges develop laterally if $c>3.45$, while the central hinge first splits if $c<3.45$ before lateral hinges can develop. In the first case the lateral hinges wander toward the center of the beam. In the second a wider central portion of the beam becomes plastic.

1. Introduction. The elasto-plastic behavior of free-free beams under dynamic loads or initial relocities has recently been studied by Bleich and Salvadori ${ }^{1}$, who obtained expressions for the elasto-plastic displacements and the plastic deformations in terms of infinite series expansions of normal modes.

Bleich and Salvadori showed that upper bounds for the plastic angle developed at the center of a free-free beam under symmetrical loads or velocities may be obtained by considering the beam as a rigid-plastic body (as suggested by Prager for concentrated dynamic loads). These bounds are good approximations of the true plastic angle whenever the dynamic loads or initial velocities are so high as to produce bending moments several times larger than the capacity moment. Another approximation of the plastic angle may be ohtained by freezing the beam into a rigid-plastic body at the time when the first hinge is developed. In the case of initial velocities this approximation is often a lower bound of the plastic angle.

Lee and Symonds ${ }^{2}$ obtained upper bounds for the plastic angle and studied the successive development of plastic hinges in rigid-plastic, free-free beams acted upon by a normal concentrated dynamic load $P$ at the middle point. They proved that, after the appearance of a central plastic hinge, two additional hinges develop laterally, which, as the load increases, wander toward the center of the beam.

In what follows the development of plastic hinges due to a symmetrical distributed load $p$ is studied by means of a concentration parameter $c$, which permits the distribution of $p$ to vary from a uniform load $p_{0}$, corresponding to $c=0$, to a concentrated load $P$, corresponding to $c=\infty$.

2. Rigid-plastic beams. A rigid-plastic beam is an idealized beam of infinite rigidity capable of becoming plastic suddenly at a section where the bending moment due to the loads and the inertia forces reaches a given value, the so-called capacity value $M_{0}$. The material of a rigid-plastic beam has therefore a stress-strain diagram of the type indi-

${ }^{*}$ Received June 10, 1952. This paper presents results of research conducted at Columbia University under contract Nonr-266 (08) with the Office of Naval Research.

${ }^{1} \mathrm{H}$. H. Bleich and M. G. Salvadori, Vibration analysis of elasto-plastic structures, with application to impulsive motion, Office of Naval Research Project NR-360-002, Contract Nonr-266(08), Technical Report No. 1, (1952).

${ }^{2}$ E. H. Lee and P. S. Symonds, Large plastic deformations of beams under transverse impact, J. Appl. Mech. 19, 308-314 (1952). 
cated in Fig. 1. Let us consider, in particular, a rigid-plastic, free-free beam of length $2 l$ and constant mass $m$ per unit of length, acted upon by a distributed normal load $p(x, t)$ and referred to an $x$-axis with origin at its middle point (Fig. 2). Under the assumption that the load $p$ be symmetrical with respect to the origin and maximum at the origin, the bending moment will at first be maximum at 0 , since the average pressure $P /(2 l)$ and the constant inertia forces per unit of length are equal and opposite, and the moment of the pressure $p(z)-P_{i}^{\prime}(2 l)$ is maximum at 0 (Fig. 3). In this first phase

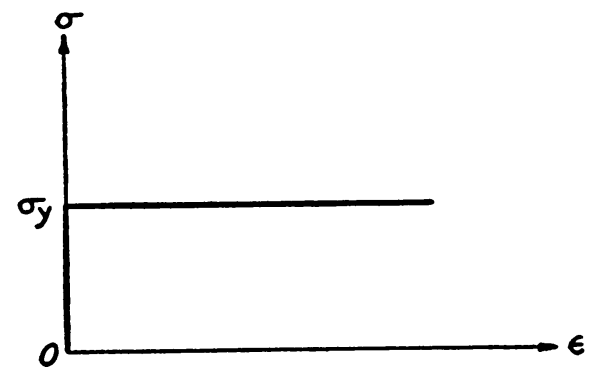

FIG. 1.

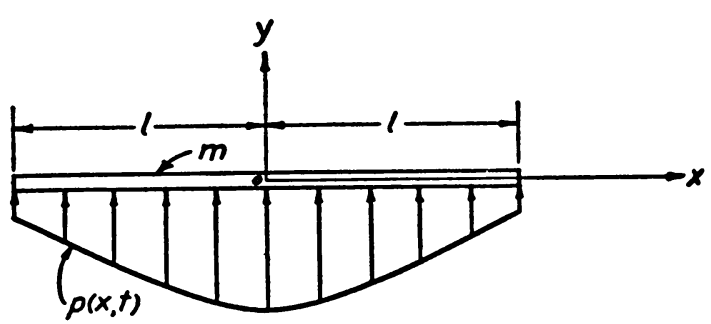

FIG. 2.

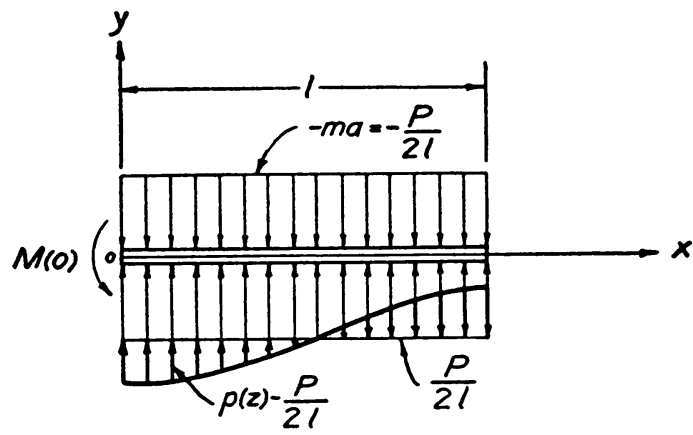

Fig. 3.

of the motion the beam translates rigidly with a ronstant acceleration $a$ in the $y$ direction and, as $p$ increases in magnitude, say linearly, there comes a moment when the

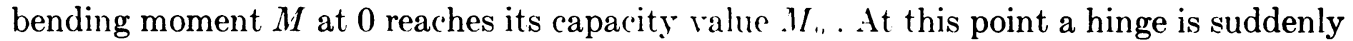
developed at 0 and each half-beam acquires an angular acceleration $-\alpha$ on top of the linear acceleration $a$. From this point on the moment of the rotational inertia forces contributes to the bending moment, which may rearh its capacity value at sections 
other than $x=\mathbf{0}$. An analysis of this second phase of the motion, limited to the development of additional lateral hinges or to the splitting of the central hinge, is contained in what follows.

3. The concentration parameter. The type of load considered is distributed symmetrically about the origin according to the exponential law:

$$
p(z)=p_{c} \frac{c}{2}(1+c z) e^{-c z} \quad(0 \leqq z \leqq 1)
$$

where:

$$
z=x / l
$$

and $c$ is a constant called the concentration parameter.

The total load on the beam due to the distribution (1) equals

$$
\begin{aligned}
P & =p_{c} l \frac{c}{2} 2 \int_{0}^{1}(1+c z) e^{-c z} d z \\
& =2 p_{c} l\left(1-e^{-c}-\frac{c}{2} e^{-c}\right)
\end{aligned}
$$

and the pressure $p_{c}$ is so chosen to make $P$ independent of $c$, so that

$$
p_{c}=\frac{P /(2 l)}{1-e^{-c}(1+c / 2)} .
$$

It is seen from Eqs. (1) and (4) that as $c$ approaches zero $p(z)$ approaches a uniform distribution $p_{0}=P /(2 l)$, while as $c$ approaches infinity $p(z)$ becomes a concentrated load $P$ applied at $x=0$. Figure 4 gives the graphs of $p(z)$ for $c=0,2,6$ and 10 .

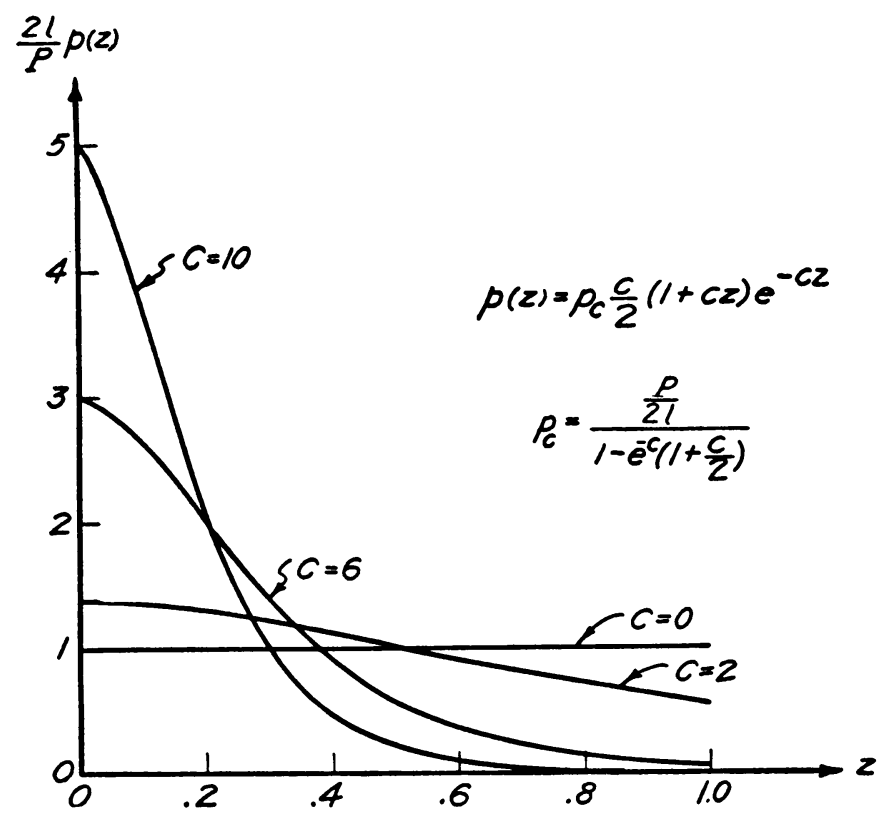

FIG. 4. 
4. The rigid translation phase. The acceleration $a$ of the beam in the $y$-direction, before the bending moment reaches its capacity value at 0 , is given by:

$$
a=\frac{P}{2 m l}=\left(p_{c} / m\right)\left[1-e^{-c}(1+c / 2)\right] .
$$

The corresponding inertia forces $-m a$ and the load $p(z)$, per unit length, create a moment $M(0)$ at the origin evaluated by the rotational equilibrium. of a half-beam about 0 (Fig. 5):

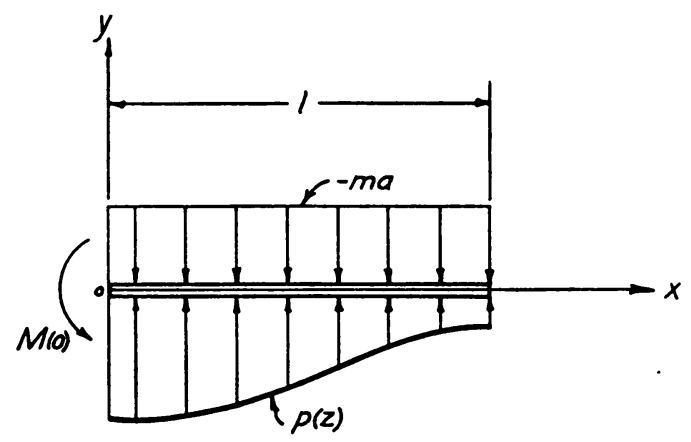

Fig. 5.

$$
\begin{aligned}
M(0) & =-p_{c} l^{2} \frac{c}{2} \int_{0}^{1}(1+c z) z e^{-c z} d z+p \frac{l^{2}}{2}\left[1-e \cdot\left(1+\frac{c}{2}\right)\right] \\
& =\frac{p_{c} l^{2}}{2}\left[1-3 / c+e^{-c}(2+c / 2+3 / c)\right]
\end{aligned}
$$

It is convenient to introduce a non-dimensional load parameter $\mu$ defined by the equation

$$
\mu=\frac{P l}{M_{0}},
$$

where $P$ is given by Eq. (3) and $M_{0}$ is the plastic capacity moment of the beam. In terms of $\mu$ the moment $M(0)$ becomes:

$$
M(0)=M_{0 \mu} \frac{1-3 / c+e^{-c}(2+c / 2+3 / c)}{4\left[1-e^{-c}(1+c / 2)\right]} .
$$

A central hinge develops and the rigid translation phase of the motion ends when $M(0)$ reaches the value $M_{0}$, that is for the value $\mu_{0}$ of $\mu$ defined by:

$$
\mu_{0}=4 \frac{1-e^{-c}(1+c / 2)}{1-3 / c+e^{-c}(2+c / 2+3 / c)} .
$$

It is seen from Eq. (9) that the value $\mu_{0}$ of $\mu$ at which the central hinge appears approaches 
4 as $c$ approaches infinity (concentrated load). As $c$ approaches zero $\mu_{0}$ becomes indeterminate, but expansion of $e^{-c}$ into a power series gives the asymptotic value

$$
\mu_{0} \doteq \frac{48}{c^{2}}-8, \quad(c \ll 1)
$$

which approaches infinity as $c$ approaches zero. The graph of $\mu_{0}$ versus $c$ appears in Fig. 6a.

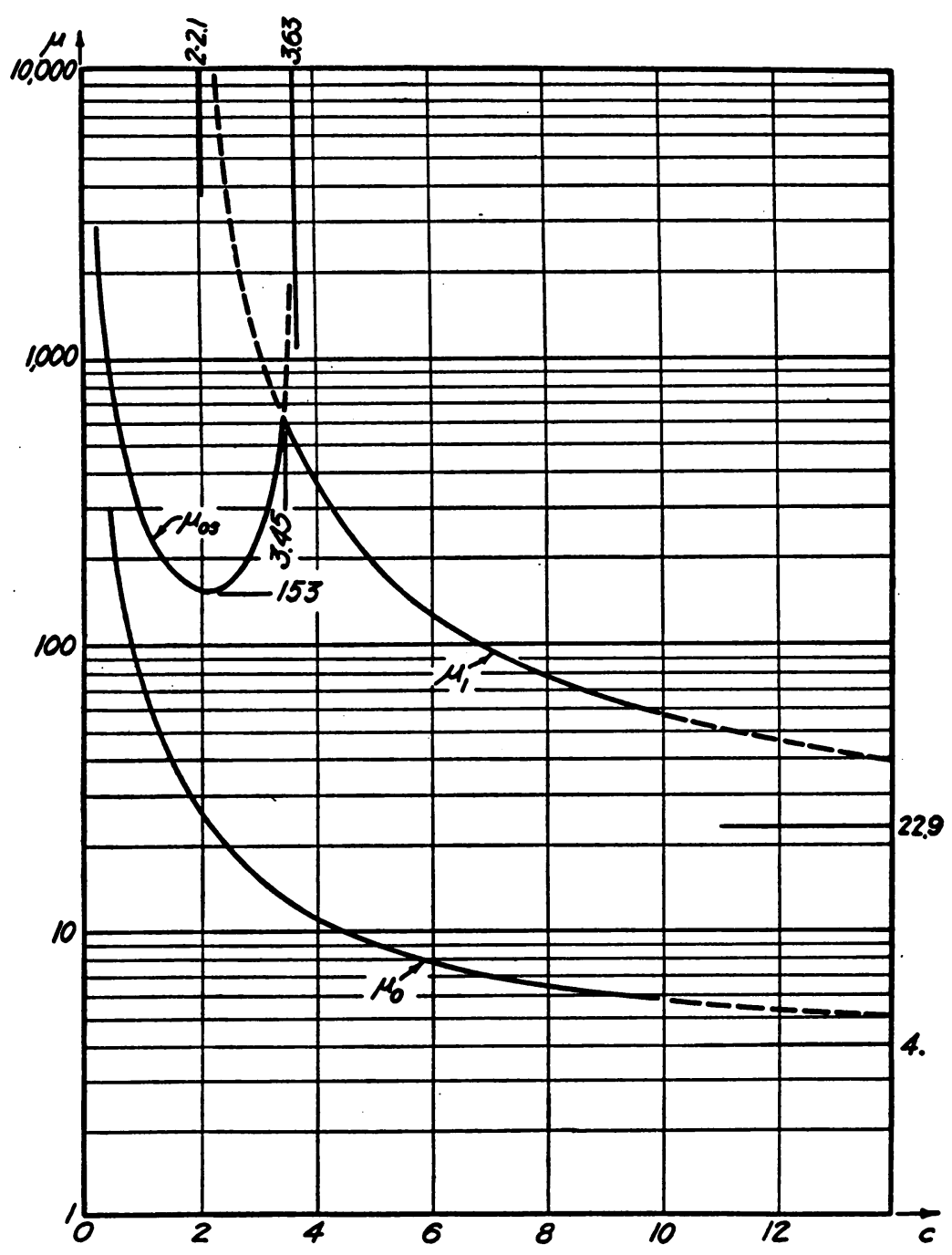

FIG. 6a.

5. The translational-rotational phase. For values of $\mu$ larger than $\mu_{0}$ the half-beams translate with an acceleration $a$ and rotate rigidly with an angular acceleration $-\alpha$, so that the acceleration of the section $x=l z$ of the beam becomes:

$$
a(z)=a-\alpha l z .
$$




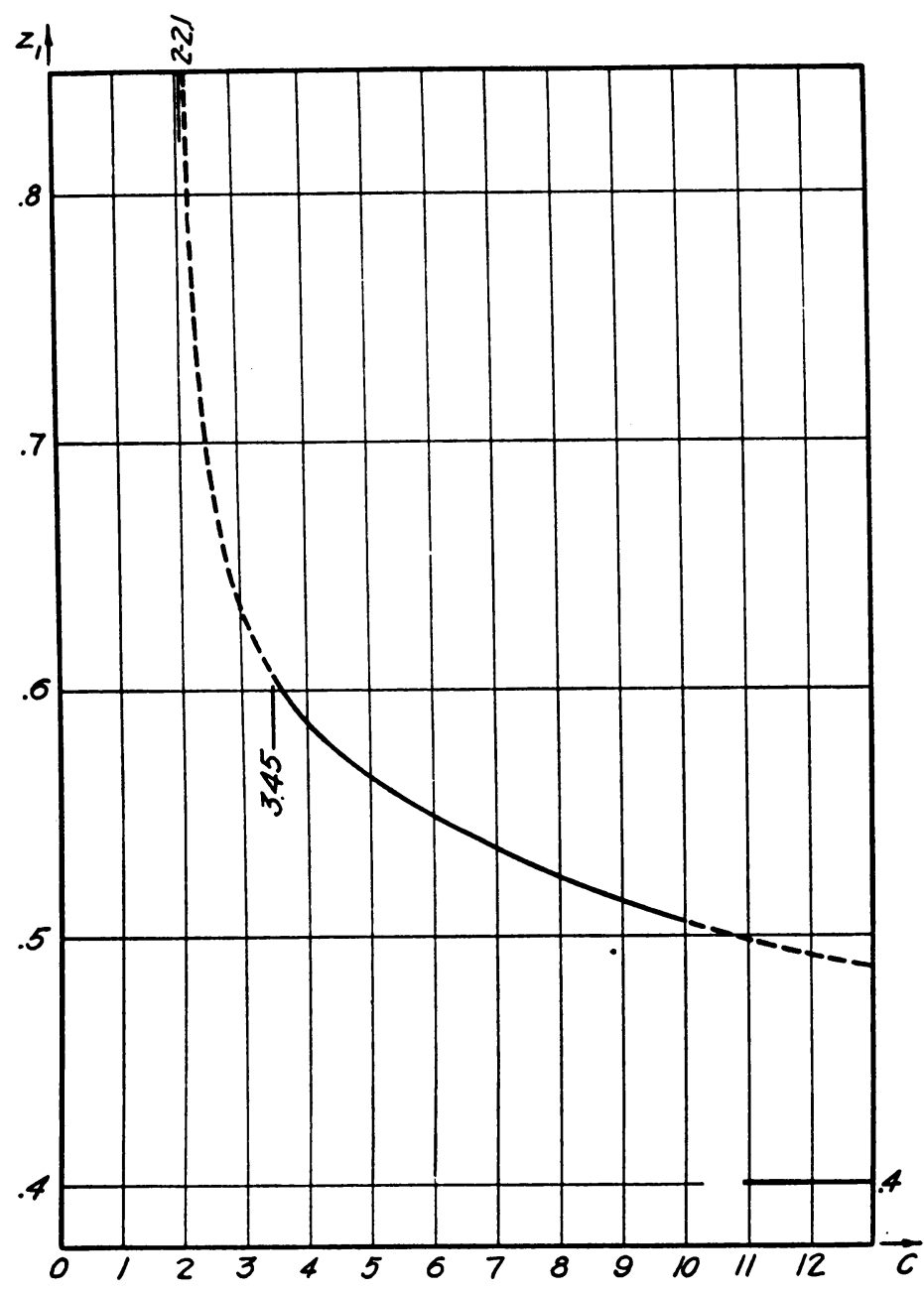

Fig. 6b.

The two unknown accelerations $a$ and $\alpha l$ are determined by applying the equations of dynamics in translation and rotation to the half-beam (Fig. 7):

$$
\begin{gathered}
P / 2-\int_{0}^{1} m a(z) d z=0 \\
M_{0}+\int_{0}^{1} p(z) z d z-m \int_{0}^{1} a(z) z d z=0 .
\end{gathered}
$$

By means of Eqs. (11), (1) and (3) these equations reduce to:

$$
\begin{gathered}
m a-\frac{1}{2} m \alpha l=p_{c}\left[1-e^{-c}(1+c / 2)\right] \\
\frac{1}{2} m a-\frac{1}{3} m \alpha l=M_{0} / l^{2}+\frac{1}{2} p_{c}\left[3 / c-e^{-c}(3+3 / c+c)\right]
\end{gathered}
$$


and give the following values of $m a$ and $m \alpha l$ :

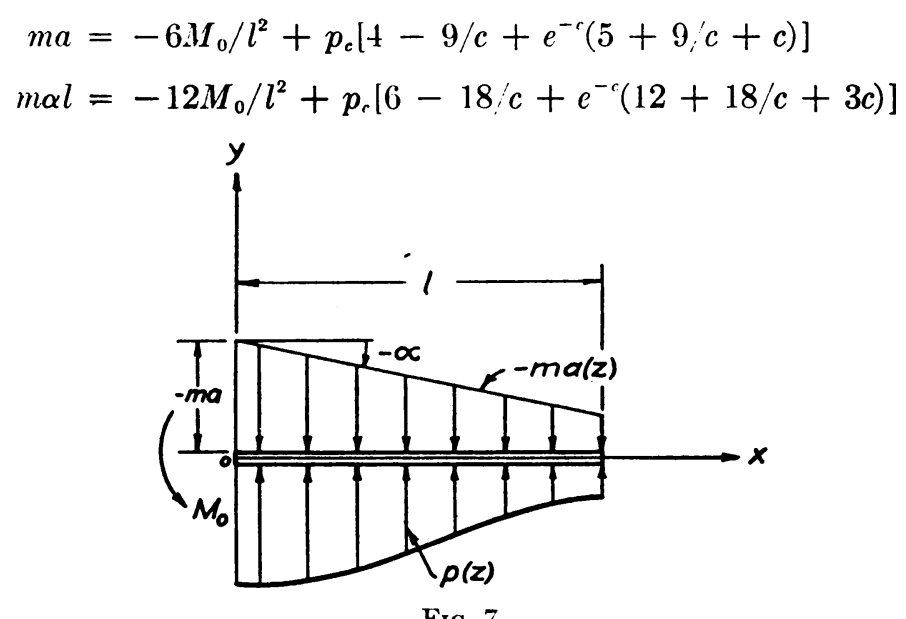

FIG. 7 .

By means of Eqs. (1), (7), (11) and (13) the bending moment at any section $x=l z$ becomes (calling $u$ the dummy variable of integration)

$$
\begin{aligned}
M(z)=l^{2} \int_{1}^{z}[p(u)-m a(u)](u-z) d u & \\
=\frac{M_{n}}{2\left[1-e^{-c}(1+c / 2)\right]}\left\{2 \left[1-e^{-c}(1\right.\right. & +c / 2)]\left(1-3 z^{2}+2 z^{3}\right) \\
& +\frac{\mu}{2}\left\{(3 / c-2 z)-e^{-c z}(z+3 / c)\right. \\
& +z^{2}\left[(4-9 / c)+e^{-c}(5+9 / c+c)\right] \\
& \left.\left.-z^{3}\left[2-6 / c+e^{-c}(4+6 / c+c)\right]\right\}\right\} .
\end{aligned}
$$

A second hinge will develop in the beam as soon as $M(z)$ has a stationary value at, say $z=z_{1} \neq 0$, and this stationary value equals the capacity moment $M_{0}$ in absolute value. Since $M(z)$ is certainly maximum at $z=0$, even after the hinge has developed at 0 , because of symmetry, the stationary value of $M$ at $z_{1}$ must be a minimum (Fig. 8)

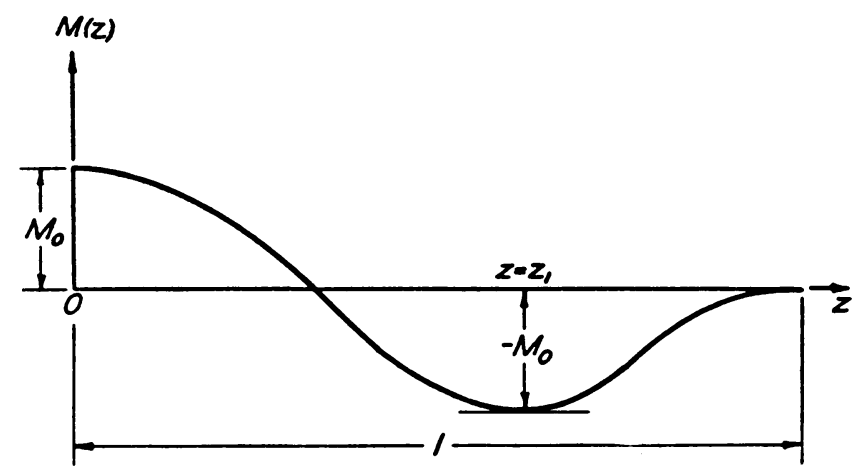

FIG. 8. 
or $M(z)$ would have a maximum larger than $M_{0}$ at another section. Hence the section $z_{1}$, at which a second hinge develops as $\mu$ increases beyond $\mu_{0}$ to a value $\mu_{1}$, is defined by the conditions

$$
\frac{M(z)}{M_{0}}=-1 \quad \frac{\partial M}{\partial z}=0,
$$

which by means of Eq. (14) become:

$$
\begin{gathered}
2\left[1-e^{-c}(1+c / 2)\right]\left(2-3 z^{2}+2 z^{3}\right)+\mu / 2\left\{(3 / c-2 z)-e^{-c z}(z+3, c)+z^{2}[(4-9 / c)\right. \\
\left.\left.+e^{-c}(5+9 / c+c)\right]-z^{3}\left[2-6 / c+e^{-c}(4+6 / c+c)\right]\right\}=0 ; \\
12\left[1-e^{-c}(1+c / 2)\right]\left(z^{2}-z\right)+\mu\left\{-\left[1-e^{-c z}\left(1+\frac{1}{2} c z\right)\right]+z[(4-9 / c)\right. \\
\left.\left.+e^{-c}(5+9 / c+c)\right]-3 z^{2}\left[(1-3 / c)+e^{-c}\left(2+3 / c+c^{\prime} 2\right)\right]\right\}=0 .
\end{gathered}
$$

Solution of the simultaneous Eqs. (16a) and (16b) for various values of $c$ gives the graphs of $\mu_{1}$ and $z_{1}$ versus $c$ plotted in Figs. $6 \mathrm{a}$ and $6 \mathrm{~b}$, respectively. It may be shown analytically that as $c$ approaches infinity $\mu_{1}$ approaches 22.9 and $z_{1}$ approaches 0.4 ; while, as $c$ decreases, the second hinge moves outward and $\mu_{1}$ increases, approaching infinity as $c$ approaches a value between 2.0 and 2.1 .

6. The splitting of the central hinge. The values of $z_{1}$ and $\mu_{1}$ obtained above for the development of a second hinge in the half-beam were predicated upon the fact that $M(z)$ would remain a maximum at $z=0$. This is guaranteed by the equation

$$
\left[\frac{\partial^{2} M}{\partial z^{2}}\right]_{z=0}<0 \text {. }
$$

If instead, as $\mu$ increases above $\mu_{0}$, the second derivative of $M$ becomes positive for values of $\mu$ such that

$$
\mu_{0}<\mu<\mu_{1},
$$

the moment $M$ would become minimum at 0 . Since this cannot happen lest $M(z)$ be greater than $M_{0}$ at another section, the central hinge splits into two symmetrical hinges which wander outward plasticizing a central portion of the beam, in which the moment has the constant value $M_{0}$. The value $\mu_{0,8}$ of $\mu$ at which the central hinge splits is therefore characterized by the condition

$$
\left[\frac{\partial^{2} M}{\partial z^{2}}\right]_{z=0}=0
$$

that is, by the equation:

$$
\left[4-9 / c-c / 2+e^{-c}(5+9 / c+c)\right] \mu+6\left[-2+e^{-c}(2+c)\right]=0,
$$

from which:

$$
\mu_{0,8}=\frac{6 c\left[2-e^{-c}(2+c)\right]}{\left(-9+4 c-c^{2} / 2\right)+e^{-c}\left(9+5 c+c^{2}\right)} \cdot
$$

The graph of $\mu_{0.8}$ versus $c$, plotted in Fig. 6a, has a minimum for $c=2.2$, approaches infinity as $c$ approaches zero and 3.63, and crosses the graph of $\mu_{1}$ for $c=3.45$.

It is thus proved that lateral hinges will develop in the beam when $\mu=\mu_{1}$ while the central hinge remains stationary if $c>3.45$. If instead $c<3.45$, the central hinge will split, plasticizing a wider central portion of the beam, when $\mu=\mu_{0, .}$, before lateral hinges may appear. 